\title{
Articular cartilage and bone changes following treatment of focal osteochondral defects in the femoral head with polyether ether ketone implants versus cobalt chromium molybdenum alloy implants: assessment in a goat model
}

\section{Zhiguo Yuan}

Shanghai Jiao Tong University School of Medicine Affiliated Renji Hospital

Wei Zhang

Shanghai Jiao Tong University School of Medicine Affiliated Renji Hospital

\section{Xiangchao Meng}

Shanghai Jiao Tong University School of Medicine Affiliated Renji Hospital

Jue Zhang

Shanghai Jiao Tong University School of Medicine Affiliated Renji Hospital

\section{Teng TengLong}

Shanghai Jiao Tong University School of Medicine Affiliated Renji Hospital

Yaochao Zhao

Shanghai Jiao Tong University School of Medicine Affiliated Renji Hospital

\section{Chunxi Yang}

Shanghai Jiao Tong University School of Medicine Affiliated Renji Hospital

Ruixin Lin

Shanghai Jiao Tong University School of Medicine Affiliated Renji Hospital

\section{Bing Yue}

Shanghai Jiao Tong University School of Medicine Affiliated Renji Hospital

You Wang ( $\sim$ drwangyou@126.com)

Shanghai Jiao Tong University School of Medicine Affiliated Renji Hospital

\section{Research article}

Keywords: polyether ether ketone (PEEK) implant, osteochondral defects, cobalt chromium molybdenum (CoCrMo)

Posted Date: April 9th, 2020

DOl: https://doi.org/10.21203/rs.3.rs-20256/v1 
License: (c) (i) This work is licensed under a Creative Commons Attribution 4.0 International License. Read Full License 


\section{Abstract}

Objective : This study aimed to investigate the feasibility of applying defect-size polyether ether ketone (PEEK) implant for the treatment of localized osteochondral defects in the femoral head and compared it with cobalt chromium molybdenum (CoCrMo) alloy implant.

Methods : A femoral head osteochondral defect model was created in the left hips of goats $(n=12)$. Defects were randomly treated by immediate placement of a PEEK $(n=6)$ or CoCrMo implant $(n=6)$. The un-operated right hip joints served as a control. Goats were sacrificed at 12 weeks. The hip joints were evaluated by gross appearance, computed tomography (CT), and magnetic resonance imaging(MRI). Periprosthetic cartilage quality and the opposing cartilage (at the acetabular) quality were analyzed macroscopically and microscopically. Implant osseointegration was measured by micro-CT and histomorphometry.

Results : Radiography revealed that all implants had good overall placement, without loosening of the implant. The modified macroscopic articular evaluation score in the PEEK group was lower than that in the CoCrMo group $(p<0.05)$, and the histological score of the periprosthetic and acetabular cartilage in the PEEK group was lower than that in the CoCrMo group $(P<0.05)$. The mean bone-implant contact for PEEK implants was comparable with that for CoCrMo alloy implants at 12 weeks.

Conclusions : A PEEK implant for the treatment of local osteochondral defect in the femoral head demonstrated effective fixation and superior in vivo cartilage protection compared with an identical CoCrMo alloy implant.

\section{Introduction}

Localized osteochondral defects in the hip are primarily caused by steroid- or alcohol-induced osteonecrosis of the femoral head (ONFD), sports injury, and hip trauma ${ }^{1 ; 2}$. ONFD at the subchondral region of the femoral head may show progressive marrow edema, bone resorption, cartilage surface fragmentation, and collapse, leading to a large focal osteochondral defect ${ }^{3}$. If not treated properly at this stage, the defect can also damage the surrounding and even opposing acetabular cartilages, deteriorating into hip osteoarthritis ${ }^{4}$. Many osteochondral repair or regenerative strategies used in the knee, such as microfracture, osteochondral autograft, and autologous chondrocyte implantation, are technically difficult to perform for the hip joint ${ }^{5 ;}$. As a result, patients with focal osteochondral injury or defects of the femoral head without severe hip osteoarthritis are often over-treated by total hip arthroplasty at a young age ${ }^{7}$.

Commonly available brand for the partial resurfacing implant is limited, so with its clinical study. Recently, Floerkemeier et $\mathrm{al}^{8}$. reported their study on the clinical outcome of partial resurfacing hip arthroplasty with HemiCap system consisting of a screw and a head resurfacing component made of 
metal alloy. The higher elastic modulus of a metal mini-implant also contributed to periprosthetic bone resorption due to stress shielding, followed by implant loosening 9 .

Polyether ether ketone (PEEK) is a potential alternative to metal alloy used in ${ }^{10}$. It offers good mechanical strength and resistance to wear, fatigue, and most chemicals and exhibits relatively lower Young's modulus, which is closer to that of the human bone as well ${ }^{11}$. Our previous study showed that PEEK on highly cross-link polyethylene (HXLPE) implant was generally safe and feasible in in vivo animal implantation study, in terms of the histology and functions of important body organs, their serological indicators, and periprosthetic bone and soft tissues ${ }^{12}$. Although the lower elastic modulus of PEEK implant tends theoretically to reduce bone loss due to stress shielding and to mitigate cartilage degeneration near the implant region due to mechanical property mismatch with normal native cartilage compared with the metal implant as partial resurfacing device of the femoral head, as a novel bearing surface, the actual in vivo behaviors of PEEK on native cartilage articulation are unknown.

In this study, two kinds of partial resurfacing implants with PEEK and CoCrMo alloy were designed for in vivo assessments of femoral head implantation in goats. One implant is made from single or monological PEEK material with a highly polished. The other implant is made from a single CoCrMo alloy material with a highly polished curved articulate surface, with shape similar to that of cemented-type pure PEEK implant. In this study, we wanted to investigate the feasibility of applying defect-size PEEK implant for the treatment of localized osteochondral defects in the femoral head and compared it with CoCrMo alloy implant.

\section{Materials And Methods}

\section{Implants}

In this study, the implants were weight-bearing, cemented-type, single-component articular implants fabricated from either PEEK or CoCrMo alloy and designed for implantation in the femoral head of goats. The implants had a highly polished curved articulate surface with a $6.4 \mathrm{~mm}$ diameter and extended to a cylindrical stem with four grooves. The articulating surfaces of both the PEEK implant and CoCrMo implant were polished to less than $3 \mu \mathrm{m}$. (Fig. 1)

\section{Animals}

Twelve adult goats, aged $2.5 \pm 0.31$ years and weighing $60.4 \pm 6.7 \mathrm{~kg}$, were used for surgery. The animals were divided according to the choice of implants used, as follows: PEEK group $(n=6)$ and CoCrMo group $(n=6)$. The un-operated right hip joints of goats served as a control. The study was undertaken after receiving approval from the Animal Ethical Committee of the Renji Hospital, Shanghai Jiaotong University, School of Medicine (Shanghai, China).

\section{Surgical Procedure}


All animals assigned to the experimental groups underwent unilateral implantation of the device in the femoral head. Surgery was performed on the right hind limb under general anesthesia. Under sterile conditions, the limb was disinfected with iodine after shaving the wool and then draped. After the joint was dislocated, the femoral head was exposed, and a tapered defect representative of a grade IV osteochondral lesion was produced using a series of custom guides and drills. The implant was then fixed into the surgically created osteochondral defect site with bone cement fixation, using custom insertion instruments to ensure device alignment and surface continuity of the implant and articular surface (Fig. 2).

\section{Imaging evaluation}

In addition to the immediate postoperative radiographs, radiographs were taken at 12 weeks postoperative. Meanwhile, all samples undertake CT scans and MRI scans. X-ray radiographs were acquired with a digital radiography system (Definium 6000, Volume RAD, GE Healthcare), whereas CT and MR images were obtained with a 16-section multi-detector row CT scanner (GE Medical Systems, Milwaukee, WI, USA) and a 3.0T MRI scanner (Philips, Amsterdam, The Netherlands), respectively.

\section{General macroscopic articular evaluation}

Two observers (Wangketao and Liuyuxin) inspected and evaluated the hip joint of goats according to the scoring system guidelines as described by O'Driscoll et al ${ }^{13 ;} 14$. The scoring system evaluates the joint through range of motion, intra-articular fibrosis, and cartilage appearance. (Table 1).

\section{Macroscopic scoring of the articular cartilage in the femoral head and acetabulum}

According to the scoring system described by Mastbergen et al. ${ }^{13 ;} 14$, two observers evaluated the degeneration of the cartilage directly in contact with the implant and the acetabulum, as part of the hip joint but not directly in contact with the implant. The two observers were blinded to the source of the photograph, and this scoring system evaluates the cartilage surface through fibrillation and grooves (Table 2).

\section{Histology of cartilage in the femoral head and acetabulum and observation of bone-implant interface}

Cartilage tissue samples were obtained from predefined locations of the femoral head and acetabulum and fixed in 10\% buffered formalin for $48 \mathrm{~h}$, embedded in paraffin, and then cut into 7- $\mu \mathrm{m}$ sections. The sections were stained with hematoxylin and eosin and Safranin-O for cartilage evaluation. Two independent and blinded reviewers examined the sections using light microscopy and evaluated the articular cartilage using the Mankin score ${ }^{15}$.

The histology-assigned femoral head containing the implants were embedded in PMMA and sectioned along the anteroposterior axis ( $100 \mu \mathrm{m}$ thick) in a longitudinal direction through the middle of the implant using an Exakt diamond blade saw. The center section of the implant was mounted on a plastic microscope slide using cyanoacrylate and polished to a final thickness of $50 \mu \mathrm{m}$. The sections were 
etched using $0.1 \%$ formic acid and subsequently stained with Van Gieson for bone-implant interface evaluation.

\section{Micro-CT examination}

The femoral head of goats' left hip joint of the hind limb and components were examined using a microCT system (SCANCO medical AG, Barsersdorf, Zurich, Switzerland) with 43- $\mu \mathrm{m}$ axial slices. CT data were examined in the transverse and sagittal planes. Three-dimensional images were reconstructed using Geomagic Studio 10.0 software program (Research Triangle Park, Chapel Hill, NC, USA). The cancellous bone was analyzed from the peri-implant zone (removal of bone cement and prosthesis), which was selected as the region of interest (ROI). We analyzed cancellous BD, BV/CV, trabecular number (Tb.N), and trabecular thickness (Tb.Th) around the implant to assess peri-implant bone quality for evaluation of the stress shielding effect.

\section{Optical profilometry of the cartilage opposite to the implant in the acetabulum}

The surface of the cartilage opposite to the implant in the acetabulum was scanned using a 3D optical profilometer (Zygo Corp., Middlefield, CT, USA). The image resolution was $640 \times 480$ pixels. The mean of the 3D surface roughness of each sample was analyzed using image analysis software (MetroPro; Zygo Corp.). The average roughness ( $\mathrm{Ra}$ ) values were the roughness parameters used to determine the surface characteristics of the sample.

\section{Statistical analysis}

Statistical analysis was performed with one-way analysis of variance using PASW for Windows software (ver. 18.0; SPSS Inc., Chicago, IL, USA). All data were expressed as means \pm standard deviation (SD), and overall significance was set at $p<0.05$.

\section{Results}

All animals had uneventful recoveries after surgery, and during the 12-week observation period, all goats were in good health. All goats were active and able to load their limbs and move the hips without any limitations.

\section{Radiographic evaluation}

After reviewing all $\mathrm{X}$ ray and $\mathrm{CT}$ results, we find no evidence of implant fracture, insert protrusion, prosthesis loosening, or sinking at 12 weeks after surgery (Fig. 3 A, B). Moreover, the MRI results show that both the PEEK implant and CoCrMo implant had good overall placement and congruous alignment of the implant articular surface with the surrounding cartilage surface (Fig. $3 \mathrm{C}$ ).

\section{General macroscopic evaluation of the hip joint and cartilage in the femoral head and acetabulum}


Gross observation showed that the implants (both PEEK and CoCrMo implant) had good congruous alignment with the surrounding cartilage surface at 12 weeks after surgery (Fig. 4). Upon sacrifice, 12 weeks after inserting the implants, the scores of the modified macroscopic articular evaluation in the CoCrMo group had decreased significantly compared with that in the PEEK group. The mean of the modified macroscopic articular evaluation parameters was not significantly different between the PEEK group and the un-operated healthy hip joints (Fig. 5).

Macroscopically, 12 weeks after treatment of the defect, the cartilage of the peri-implant in the femoral head and opposite to the implant in the acetabulum showed mild degeneration, ranging from slightly fibrillated to fibrillated with shallow grooves. The mean of the macroscopic cartilage score in the PEEK group was lower than that in the CoCrMo group $(p<0.05)$ (Fig. 6)

\section{Histologic cartilage evaluation and osseointegration}

We analyzed the histology of the peri-implant cartilage in the femoral head and the cartilage opposite to the implant in the acetabulum using the Mankin score for sections stained with HE and Safranin $\mathrm{O}$. The scores for the amount of cartilage of the peri-implant in the femoral head showed no significant differences between the PEEK group and CoCrMo group at 12 weeks after surgery, while the Mankin score of the cartilage opposite to the implant in the acetabulum for the PEEK group was significantly improved compared with that of the CoCrMo group $(\mathrm{p}<0.05)$ (Fig. 7).

Histologic sections (containing the implant) for Van Gieson staining at 12 weeks post-operation demonstrated excellent maintenance of the host cartilage-implant interface with minimal cellular and mechanical changes present for the PEEK implant. Histologic observations of the bone-implant interface for PEEK implants were comparable with those of CoCrMo alloy implants at 12 weeks, which maybe because both PEEK and CoCrMo implants were fixed by cement.(Fig. 8)

\section{Micro-CT analysis}

Moreover, 2D images of the femoral head and 3D images of new bones with peripheral implant (volume of interest, VOI) were reconstructed. Micro-CT analysis revealed the BD, BV/TV, Tb.N, and Tb.Th of the $\mathrm{VOI}$. The BD of the femoral head in both groups decreased at 12 weeks compared with that in the control group, while no significant difference was found between the PEEK group and CoCrMo group. The BV/TV of the femoral head in the CoCrMo group decreased significantly compared with that of the control group $(p<0.05)$, while no significant difference was found between the PEEK group and CoCrMo group. The Tb.N of the region of interest in both the PEEK group and CoCrMo group was decreased compared with that of controls, and the Tb.N of the CoCrMo group was lower than that of the PEEK group. The Tb.Th of the VOI in both the PEEK group and CoCrMo group was decreased compared with that in the control group, while no significant difference was found between the PEEK group and CoCrMo group (Fig. 9).

\section{Optical profile analysis of cartilage opposite to the implant in the acetabulum}


We utilized an optical profilometer to analyze the surface roughness of the cartilage opposite to the implant in the acetabulum. The results show that the surface roughness of the cartilage in the acetabulum for the CoCrMo group is higher than that for the PEEK group, which is comparable with that of the control group (Fig. 10).

\section{Discussion}

This study demonstrated that a PEEK hemiarthroplasty implant material was superior to a CoCrMo alloy implant. Moreover, at 12 weeks after surgery, there was less cartilage degeneration (such as surface cartilage wear, degradation, and cellular change) in the cartilage peri-implant in the femoral head and cartilage opposite to the implant in the acetabulum for the PEEK implants compared with that for the CoCrMo alloy implants. These findings were demonstrated both macroscopically and microscopically. Macroscopic joint and cartilage evaluation demonstrated increased joint and cartilage degenerations, while the degeneration caused by CoCrMo alloy implant was more severe than that by PEEK implants. Moreover, similar results were seen in the macroscopic cartilage scores, where the acetabulum, directly articulating surface against the implants, was significantly degenerated. This was also confirmed by the histology of cartilage explants from the peri-implant region and the area opposite to the implant in the acetabulum. Meanwhile, the surface roughness of the cartilage in the acetabulum was higher in the CoCrMo group than in the PEEK group.

In line with the macroscopic and microscopic results, cartilage degradation in the hips resulting from the treatment effects of the CoCrMo alloy implants was higher than that the treated effects of the PEEK implants. However, the peri-implant bone mass or bone density for the PEEK implant was not better than that for the CoCrMo alloy implants, which maybe because the implants were fixed by cement. In this study, the BD of the region of interest in both groups decreased at 12 weeks compared with that in the control group, while no significant difference was found between the PEEK group and CoCrMo group, whereras the Tb.N of the region of interest in both the PEEK group and CoCrMo group was decreased compared with that of controls, and the Tb.N of the CoCrMo group was lower than that of the PEEK group, and we guess that the priority of the peri-implant bone preservation due to less stress shielding of low Young's modulus of the PEEK implants was compensated by the cement fixation effect. In our previous study, we also found that the BD of the peri-implant decreased in the cemented implant ${ }^{16}$. And in some cemented total knee arthroplasty, the density of bone peri cemented stem in the tibial metaphysis decreased ${ }^{17 ; 18}$. However, in an uncemented study, comparing the results of untreated critical-size cartilage defects in the medial femoral condyle of goats with defects treated with oxidized zirconium small hemiarthroplasty implants after 52 weeks, the implants showed good osseointegration ${ }^{19 ;} 20$. So we infer that the PEEK implant may have better osseointegration and higher bone-implant contact compare to metal implant in an uncemented condition, as its similar biomechanical property to bone, which means less stress shielding.

In this study, the implants in the femoral head was shown to have profound effects on the degeneration of the peri-implant cartilage in the femoral head and the opposite cartilage in the acetabulum, with flush 
placement of the PEEK implants causing less degeneration and better integration with surrounding cartilage compared with those caused by CoCrMo alloy implants. PEEK implants mediated less degeneration compared with CoCrMo alloy implants because of its lower elastic modulus, similar to the bone. This was also confirmed by Cook et al., who evaluated the degeneration of acetabular cartilage following implantation of proximal femoral hemiarthroplasties in dogs $^{21}$. Results indicated that the native acetabulum in articulation with pyrolytic carbon implants experienced a lesser degree of joint degeneration, and there was significantly greater probability of cartilage survival with articulation against the pyrolytic carbon implant than the metal alloy implants. Moreover, PEEK materials has been shown to have superior qualities for use in partial and total joint replacements, as its elastic modulus is similar to the bone, which reduces stress shielding 22 . In the present study, based on the direct comparison of PEEK implants with identical metal alloy implants, we hypothesized that the improved performance of PEEK devices results from the reduced material elastic modulus, as well as lower surface energy and the nonadhesive nature of the PEEK surface.

This study has some limitations. First, in the animal model, the implants were placed in a joint that was not truly arthritic. the implants were placed in a joint at the same time that the defect was made. Second, this study did not include a control group with osteochondral defects that were left untreated. Third, the follow-up time was only 12 weeks, which may be not long enough. Finally, although the PEEK implant show significantly advantage in reducing degeneration of the cartilage compared to metal implant, the mechanical property of the PEEK material was still higher than the native cartilage.

\section{Abbreviations}

PEEK: polyether ether ketone; CoCrMo: cobalt chromium molybdenum $囚 \mathrm{CT}$ : computed tomography; MRI: magnetic resonance imaging $₫ \mathrm{ONFD}$ : osteonecrosis of the femoral head囚HXLPE: highly cross-link polyethylene; VOI: volume of interest; Tb.N: trabecular number; Tb.Th: trabecular thickness; ROI: region of interest.

\section{Declarations}

\section{Ethics approval}

This study received approval from the Animal Ethical Committee of the Renji Hospital, Shanghai Jiaotong University, School of Medicine for all animal care and procedures.

\section{Consent for publication}

Not applicable

\section{Availability of data and materials}


The datasets during and/or analyzed during the current study are available from the corresponding author on reasonable request.

\section{Competing interests}

The authors declare that they have no competing interests.

\section{Funding}

This study was supported by the National Key R\&D Program of China (Grant No. 2016YFC1101802). The funders had no role in the study design, data collection and analysis, decision to publish, or preparation of the manuscript.

\section{Authors' contributions}

All authors participated in the surgery, contributed to the manuscript preparation, and approved the final manuscript.

\section{Acknowledgements}

we thank Zhonglin Zhu from Jiangsu Okani Medical Technology Co., Ltd. (Soochow, JS, China) for providing technical support and discussing the experimental strategy.

\section{References}

1. Klennert BJ, Ellis BJ, Maak TG, et al. The mechanics of focal chondral defects in the hip. J Biomech. 2017;52:31-7.

2. Fontana A, Mancini D, Gironi A, et al. Hip osteochondral lesions: arthroscopic evaluation. Hip Int. 2016;26(Suppl 1):17-22.

3. Piuzzi NS, Anis HK, Muschler GF. Osteonecrosis of the femoral head with subchondral collapse. Cleve Clin J Med. 2019;86:511-2.

4. Wei B, Gu Q, Li D, et al. Mild degenerative changes of hip cartilage in elderly patients: an available sample representative of early osteoarthritis. Int J Clin Exp Pathol. 2014;7:6493-503.

5. Nam D, Shindle MK, Buly RL, et al. Traumatic osteochondral injury of the femoral head treated by mosaicplasty: a report of two cases. HSS J. 2010;6:228-34.

6. Oladeji LO, Cook JL, Stannard JP, et al. Large fresh osteochondral allografts for the hip: growing the evidence. Hip Int. 2018;28:284-90.

7. Shon WY, Park BY, R RN, et al. Total Hip Arthroplasty: Past, Present, and Future. What Has Been Achieved? Hip Pelvis. 2019;31:179-89.

8. Floerkemeier T, Budde $\mathrm{S}$, Wirries $\mathrm{N}$, et al. The outcome of the partial resurfacing arthroplasty of the hip shows high numbers of failures and conversion to total arthroplasty. Int Orthop. 2017;41:2001-8. 
9. Chang JZ, Chen YJ, Tung YY, et al. Effects of thread depth, taper shape, and taper length on the mechanical properties of mini-implants. Am J Orthod Dentofacial Orthop. 2012;141:279-88.

10. Vaishya R, Agarwal AK, Tiwari M, et al. Medical textiles in orthopedics: An overview. J Clin Orthop Trauma. 2018;9:26-33.

11. Almasi D, Iqbal N, Sadeghi M, et al. 2016. Preparation Methods for Improving PEEK's Bioactivity for Orthopedic and Dental Application: A Review. Int J Biomater 2016:8202653.

12. Du Z, Zhu Z, Wang Y. The degree of peri-implant osteolysis induced by PEEK, CoCrMo, and HXLPE wear particles: a study based on a porous Ti6Al4V implant in a rabbit model. J Orthop Surg Res. 2018;13:23.

13. O'Driscoll SW, Keeley FW, Salter RB. Durability of regenerated articular cartilage produced by free autogenous periosteal grafts in major full-thickness defects in joint surfaces under the influence of continuous passive motion. A follow-up report at one year. J Bone Joint Surg Am. 1988;70:595-606.

14. O'Driscoll SW, Keeley FW, Salter RB. The chondrogenic potential of free autogenous periosteal grafts for biological resurfacing of major full-thickness defects in joint surfaces under the influence of continuous passive motion. An experimental investigation in the rabbit. J Bone Joint Surg Am. 1986;68:1017-35.

15. van der Sluijs JA, Geesink RG, van der Linden AJ, et al. The reliability of the Mankin score for osteoarthritis. J Orthop Res. 1992;10:58-61.

16. Du Z, Zhu Z, Yue B, et al. Feasibility and Safety of a Cemented PEEK-on-PE Knee Replacement in a Goat Model: A Preliminary Study. Artif Organs. 2018;42:E204-14.

17. Lonner JH, Klotz M, Levitz C, et al. Changes in bone density after cemented total knee arthroplasty: influence of stem design. J Arthroplasty. 2001;16:107-11.

18. Hernandez-Vaquero D, Garcia-Sandoval MA, Fernandez-Carreira JM, et al. Influence of the tibial stem design on bone density after cemented total knee arthroplasty: a prospective seven-year follow-up study. Int Orthop. 2008;32:47-51.

19. Custers RJ, Saris DB, Dhert WJ, et al. Articular cartilage degeneration following the treatment of focal cartilage defects with ceramic metal implants and compared with microfracture. J Bone Joint Surg Am. 2009;91:900-10.

20. Custers RJ, Dhert WJ, Saris DB, et al. Cartilage degeneration in the goat knee caused by treating localized cartilage defects with metal implants. Osteoarthritis Cartilage. 2010;18:377-88.

21. Cook SD, Weinstein AM, Klawitter JJ, et al. Quantitative histologic evaluation of LTI carbon, carboncoated aluminum oxide and uncoated aluminum oxide dental implants. J Biomed Mater Res. 1983;17:519-38.

22. Panayotov IV, Orti V, Cuisinier F, et al. Polyetheretherketone (PEEK) for medical applications. J Mater Sci Mater Med. 2016;27:118.

\section{Tables}


Table I

Modified Macroscopic Articular Evaluation Parameters

\begin{tabular}{|l|l|}
\hline Characteristics & Score \\
\hline 1. Range of motion & \\
\hline a. Full & 2 \\
\hline b. $<20$ & 1 \\
\hline c. $>20$ & 0 \\
\hline 2. Intra-articular fibrosis & \\
\hline a. None & 2 \\
\hline b. Minor & 1 \\
\hline c. Major & 0 \\
\hline 3. Appearance & \\
\hline a. Translucent & 2 \\
\hline b. Opaque & 1 \\
\hline c. Discolored/irregular & 0 \\
\hline Total & $0-6$ \\
\hline
\end{tabular}

Table II

Macroscopic cartilage score as described by Mastbergen et al.

\begin{tabular}{|l|c|}
\hline Femoral head & \\
\hline Smooth surface & 0 \\
\hline Slightly fibrillated & 1 \\
\hline Fibrillated with shallow grooves & 2 \\
\hline Deep sharp grooves & 3 \\
\hline Deep sharp grooves with surrounding damage & 4 \\
\hline Acetabulum & \\
\hline Smooth surface & 0 \\
\hline Slightly fibrillated & 1 \\
\hline Fibrillated with shallow grooves & 2 \\
\hline Deep sharp grooves & 3 \\
\hline Deep sharp grooves with surrounding damage & 4 \\
\hline & \\
\hline
\end{tabular}

\section{Figures}


A

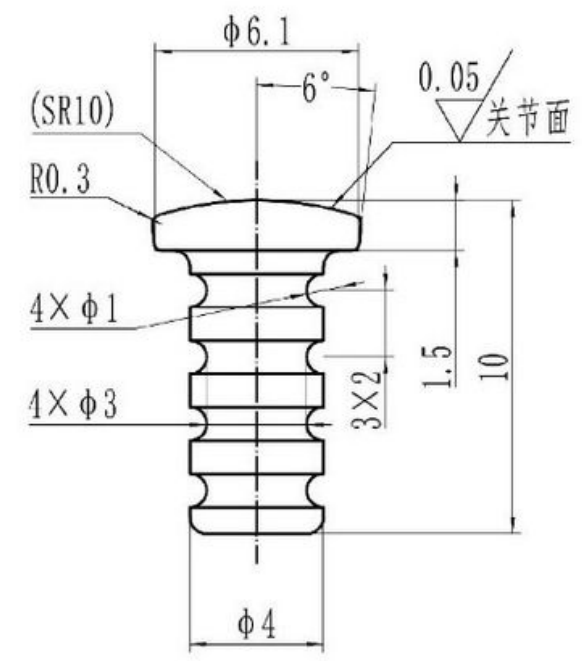

B
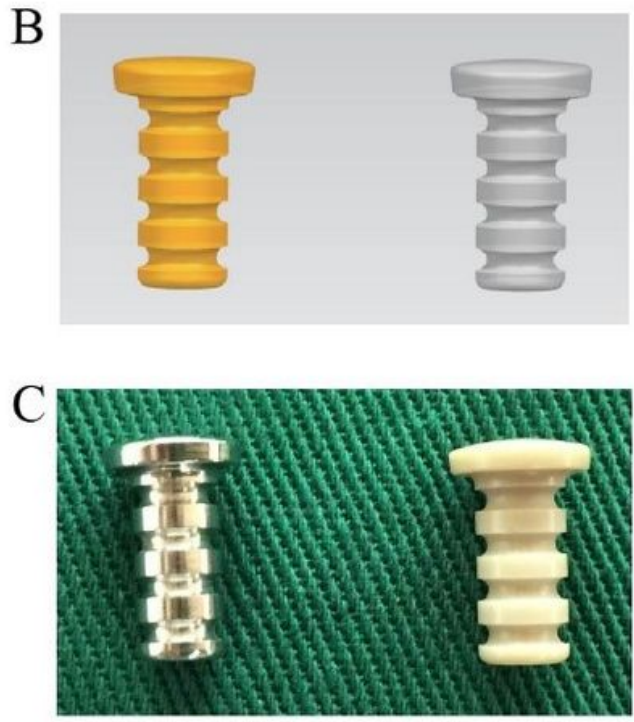

CoCrMo

PEEK
$\mathrm{D}$

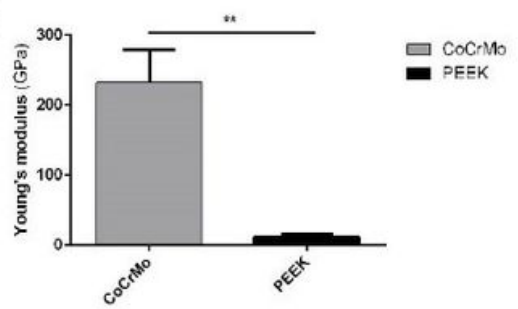

E

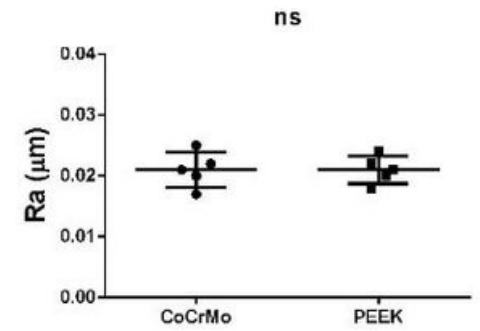

Figure 1

Characteristics of the implants. A. Technical drawing of the implants. B. Model figures of the implants. C. Femoral head tack implant made of CoCrMo (left) and PEEK (right). D. Young modulus of the implants. E. $\mathrm{Ra}$ of the implants' surfaces was polished to less than $0.03 \mu \mathrm{m}$. CoCrMo, cobalt chromium molybdenum; PEEK, polyether ether ketone implant 


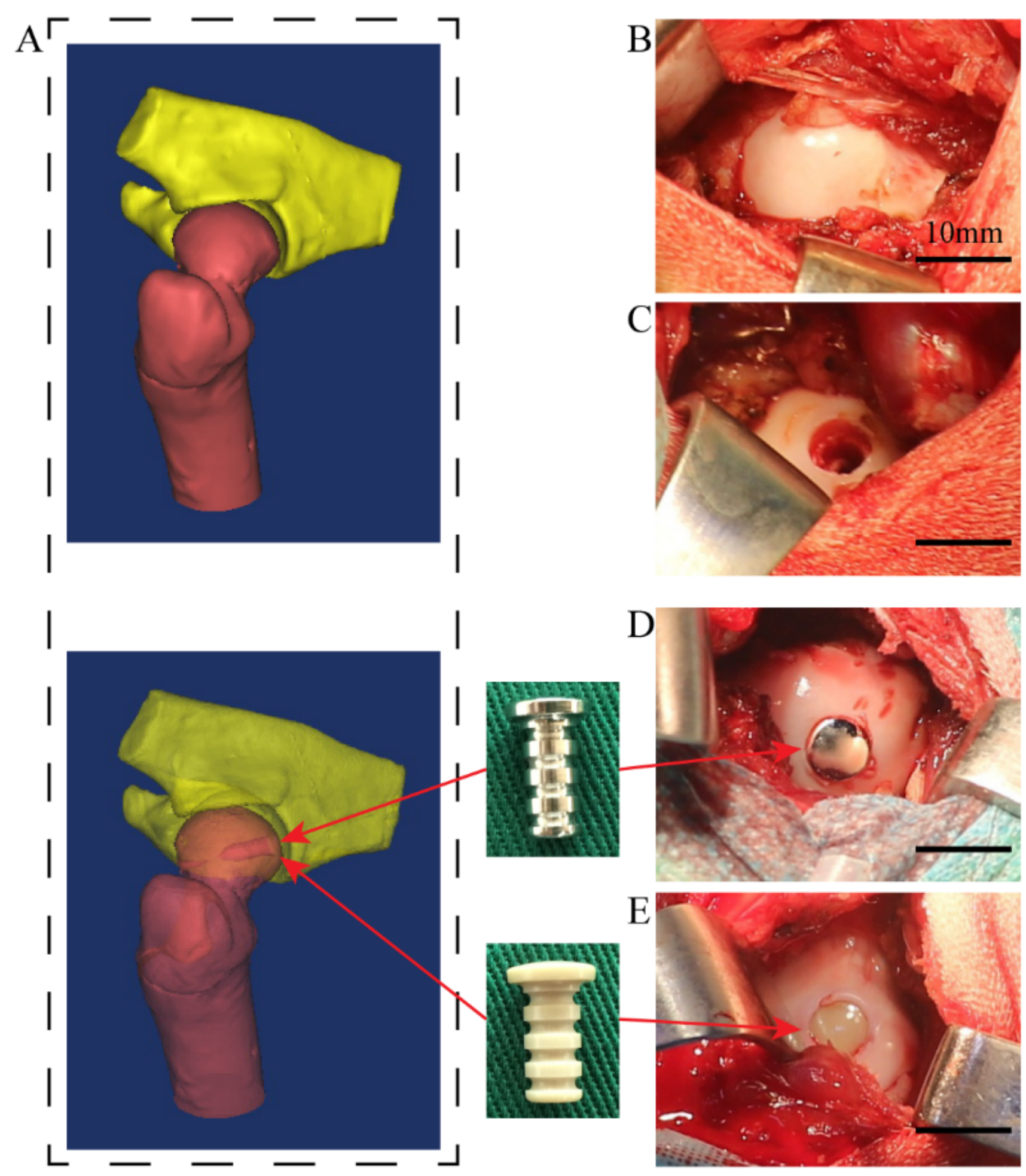

Figure 2

Operation strategy of the implants. A. 3D model of implant position B. Native femoral head of a goat. C. Osteochondral defect in the femoral head of a goat. D. Implantation of CoCrMo and PEEK implants in the femoral head of the goat. E. Implantation of PEEK implants in the femoral head of a goat. 3D, three dimensional; CoCrMo, cobalt chromium molybdenum; PEEK, polyether ether ketone implant 

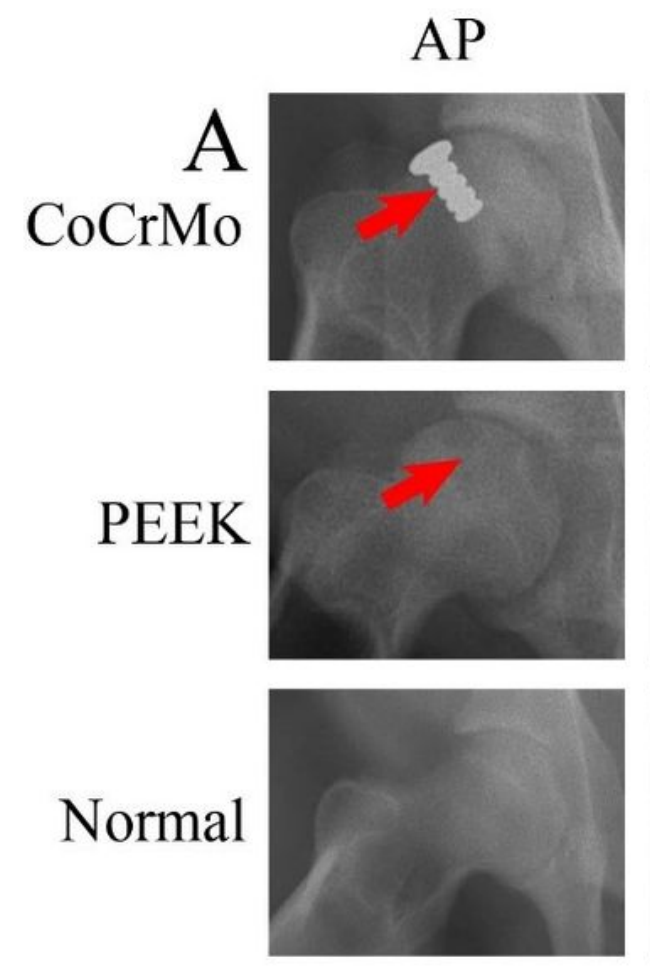

\section{Coronal}
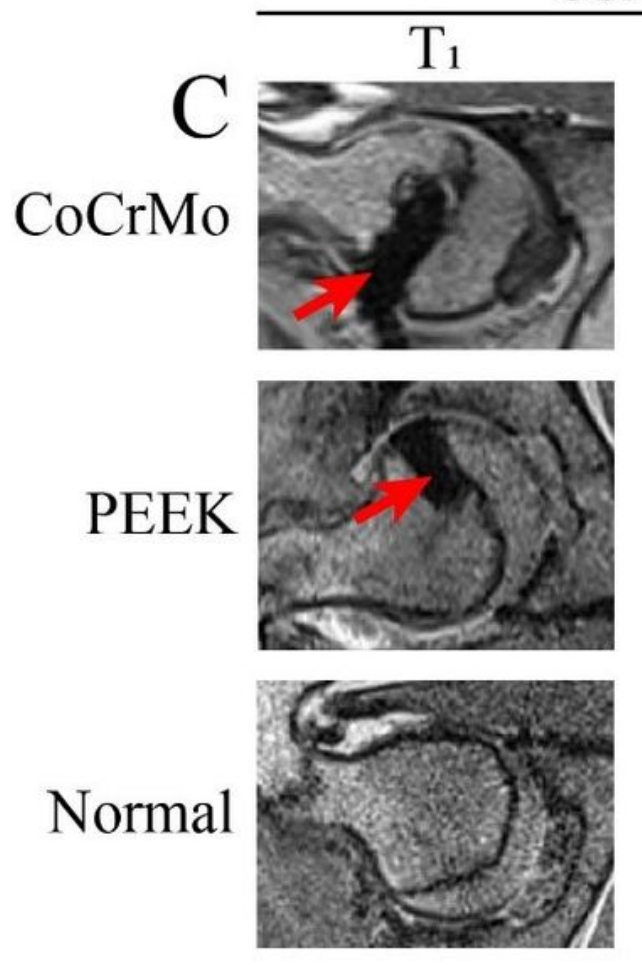

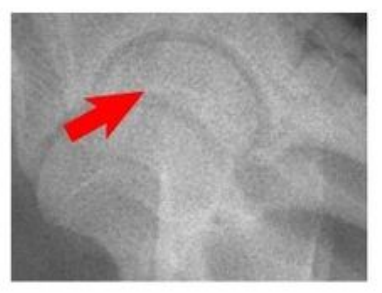

Lateral
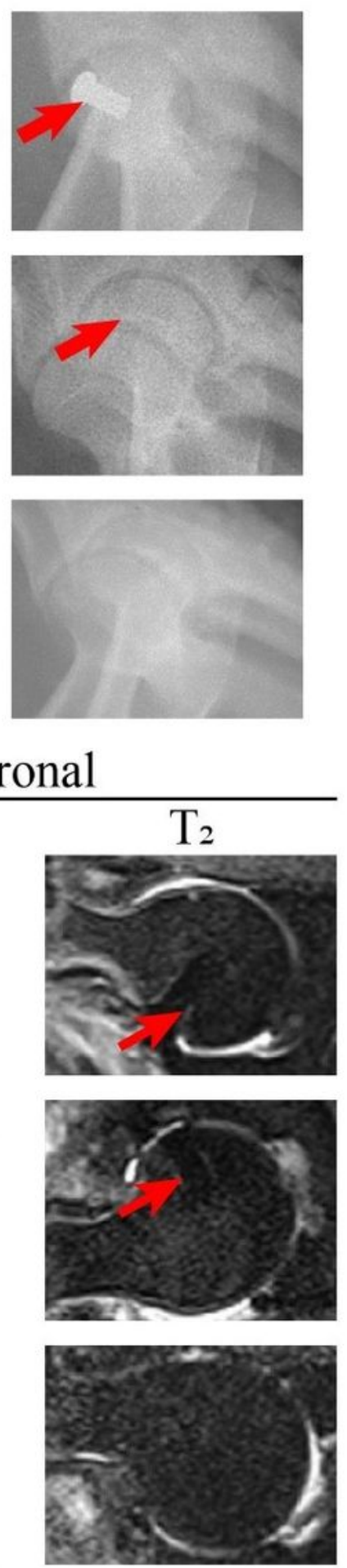

Coronal

B
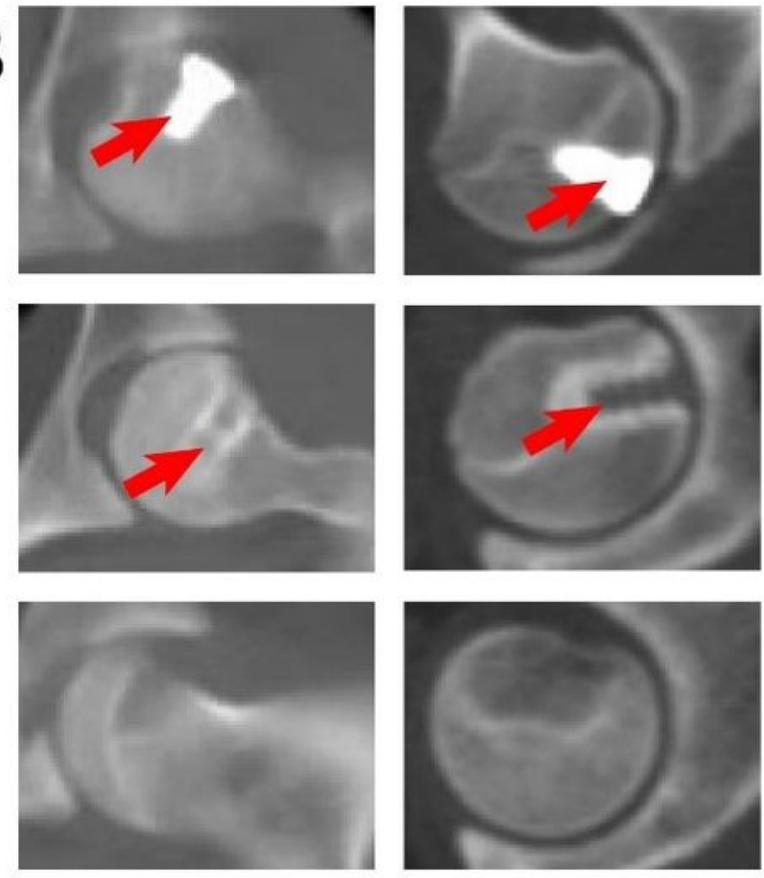

Sagittal
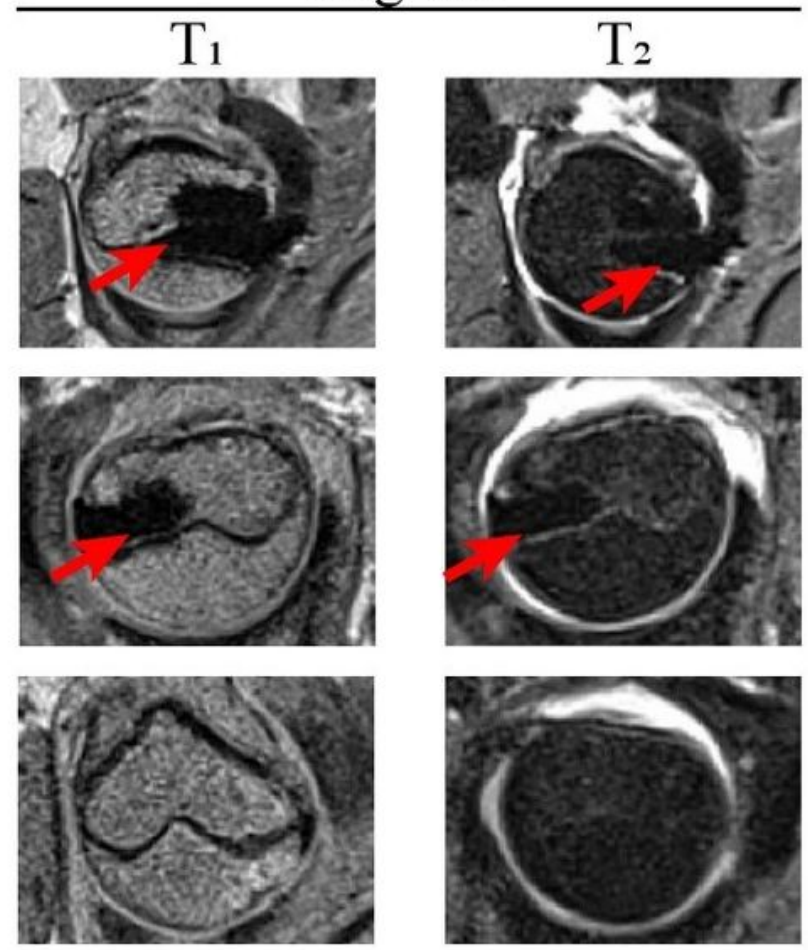

Sagittal
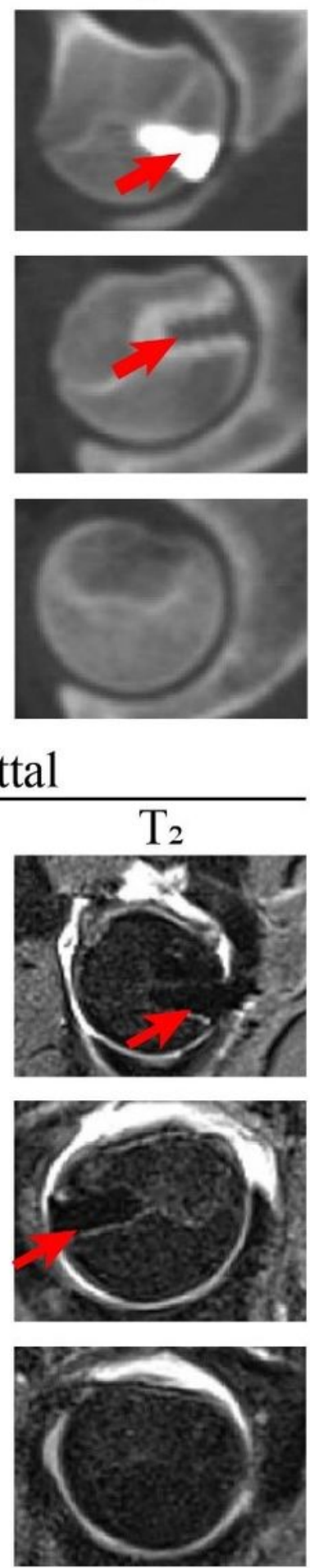

\section{Figure 3}

X-ray, CT, and MR images of the operated hip joint. A. X-ray radiographs of the CoCrMo, PEEK, and native groups. B. CT images of the femoral head in the CoCrMo, PEEK, and native groups. C. MR images of the femoral head in the CoCrMo, PEEK, and native groups. The red arrows indicate the position of the implants. CoCrMo, cobalt-chromium-molybdenum alloy; CT, computed tomography, MRI, magnetic resonance imaging; PEEK, polyether ether ketone; Ra, mean surface roughness 

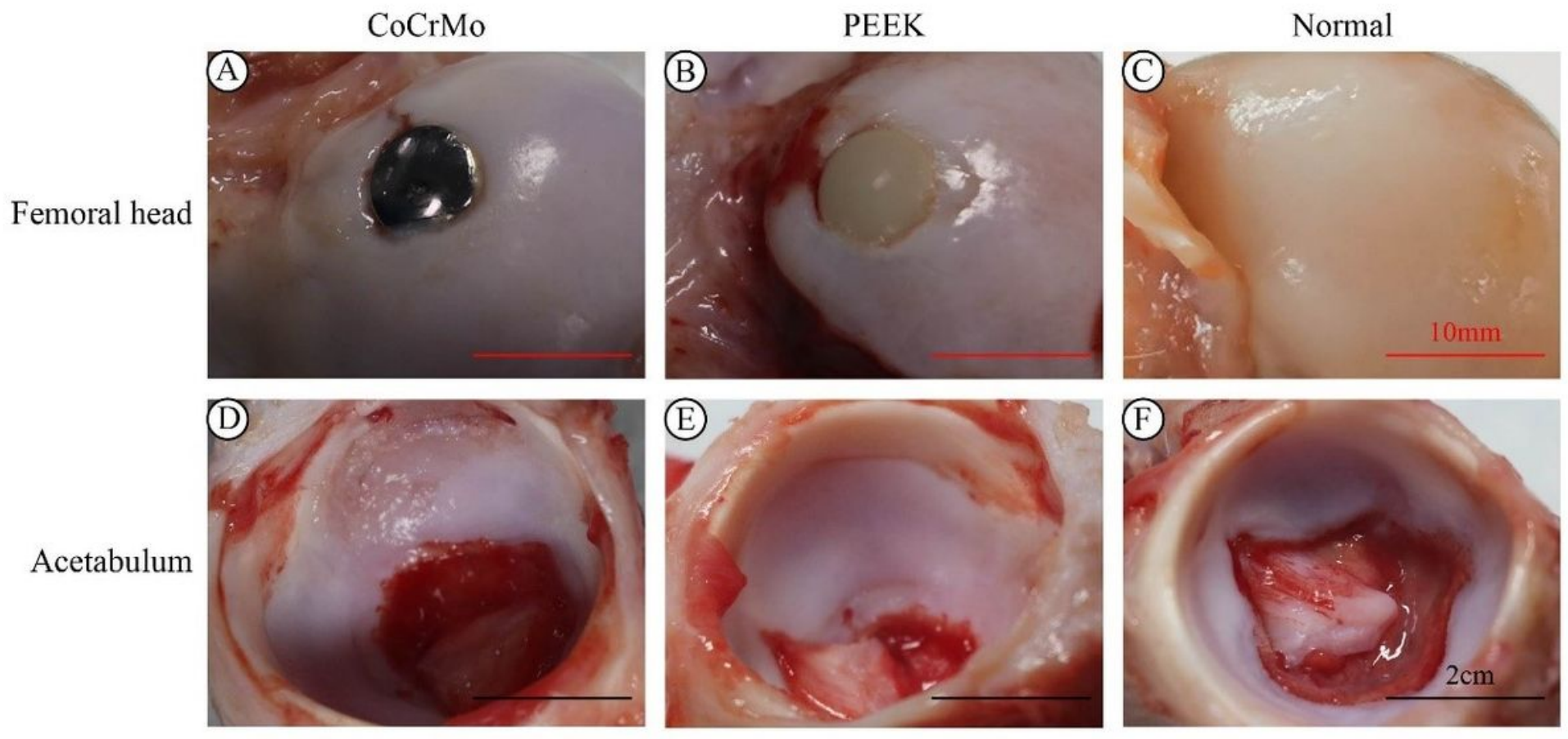

\section{Figure 4}

Examples of the femoral head and acetabulum after 12 weeks of follow-up. A. CoCrMo implant. The implant is surrounded by relatively healthy cartilage. B. PEEK implant. The implant is surrounded by relatively healthy cartilage. C. Native femoral head. D. Macroscopical image of the acetabulum in the CoCrMo group. E. Macroscopical image of the acetabulum in the PEEK group. F. Macroscopical image of the acetabulum in the native group. CoCrMo, cobalt chromium molybdenum; PEEK, polyether ether ketone implant 


\section{Macroscopic Articular Evaluation Parameters}

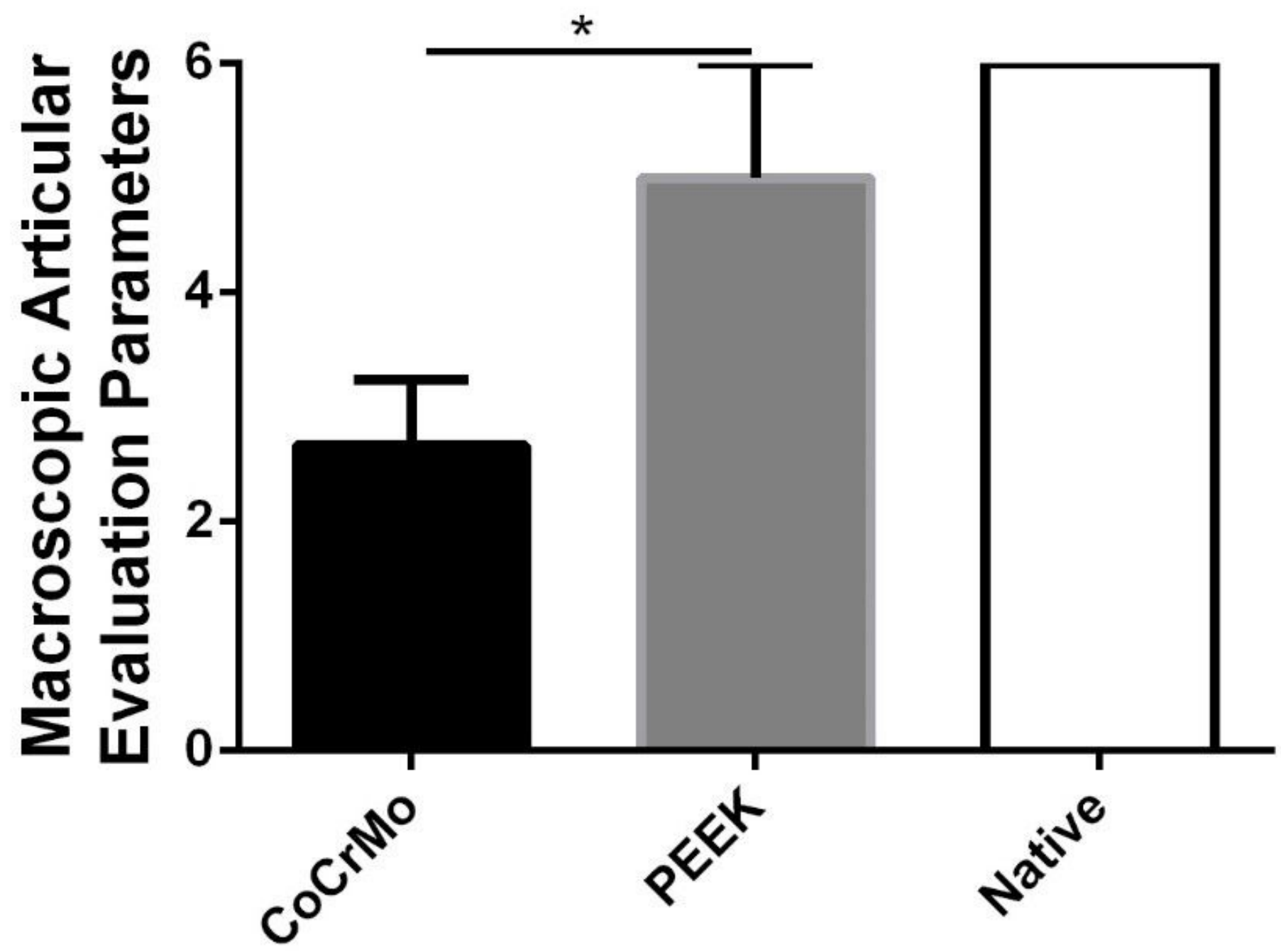

Figure 5

The modified Macroscopic Articular Evaluation Parameters. A score of 0 represents a severely degenerated, fibrillated, and fixated hip joint, whereas a score of 6 represents a healthy joint. $\left({ }^{\star} p<0.05\right)$ 


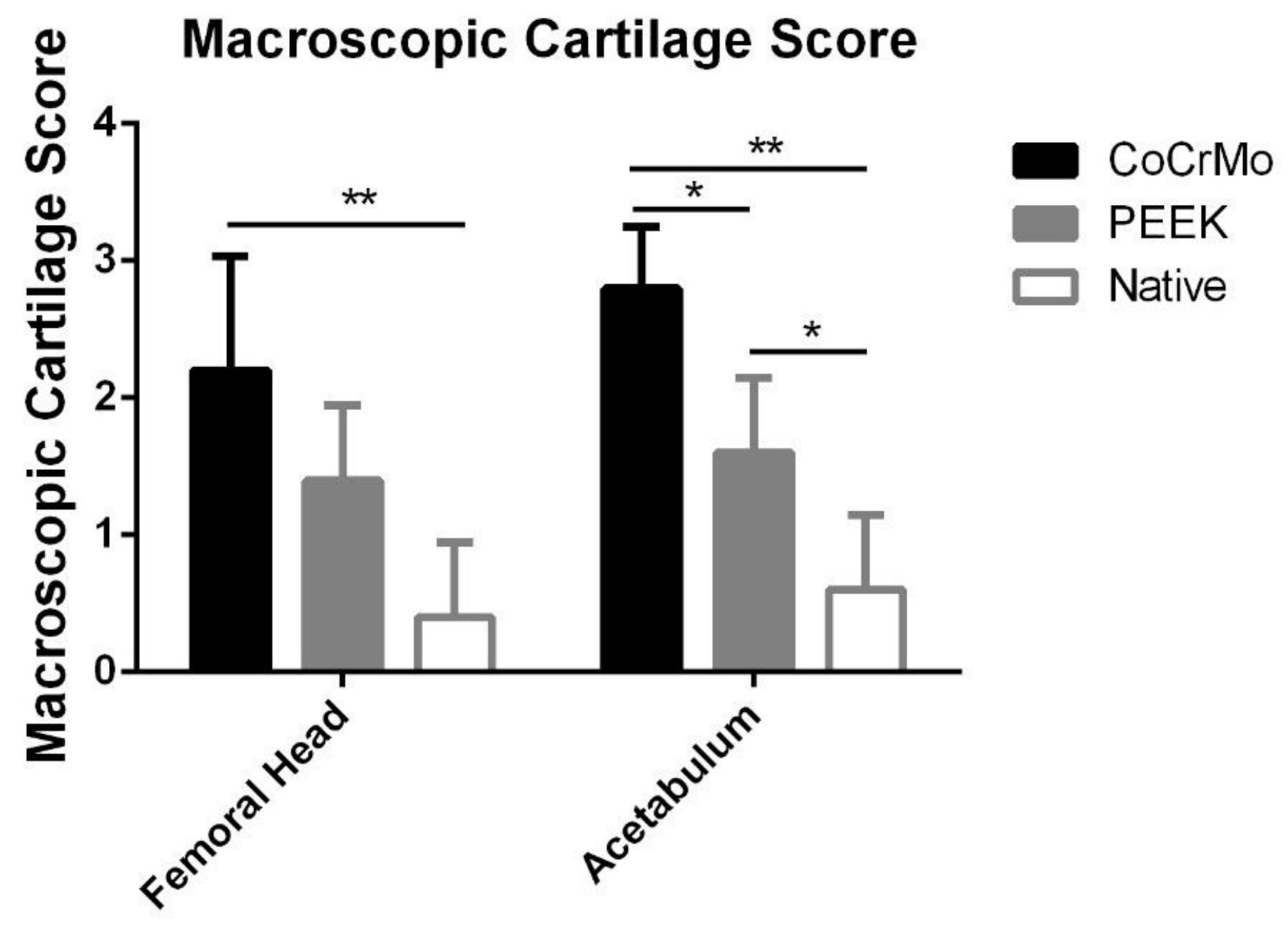

Figure 6

Macroscopic cartilage score 12 weeks after surgery. The scores of the CoCrMo, PEEK, and native groups are represented respectively by the black, gray, and white bars. A higher score indicates that the periimplant cartilage (femoral head) and the cartilage opposite to the implant (acetabulum) degenerated, ranging from fibrillated with shallow grooves to deep sharp grooves. CoCrMo, cobalt chromium molybdenum; PEEK, polyether ether ketone implant 

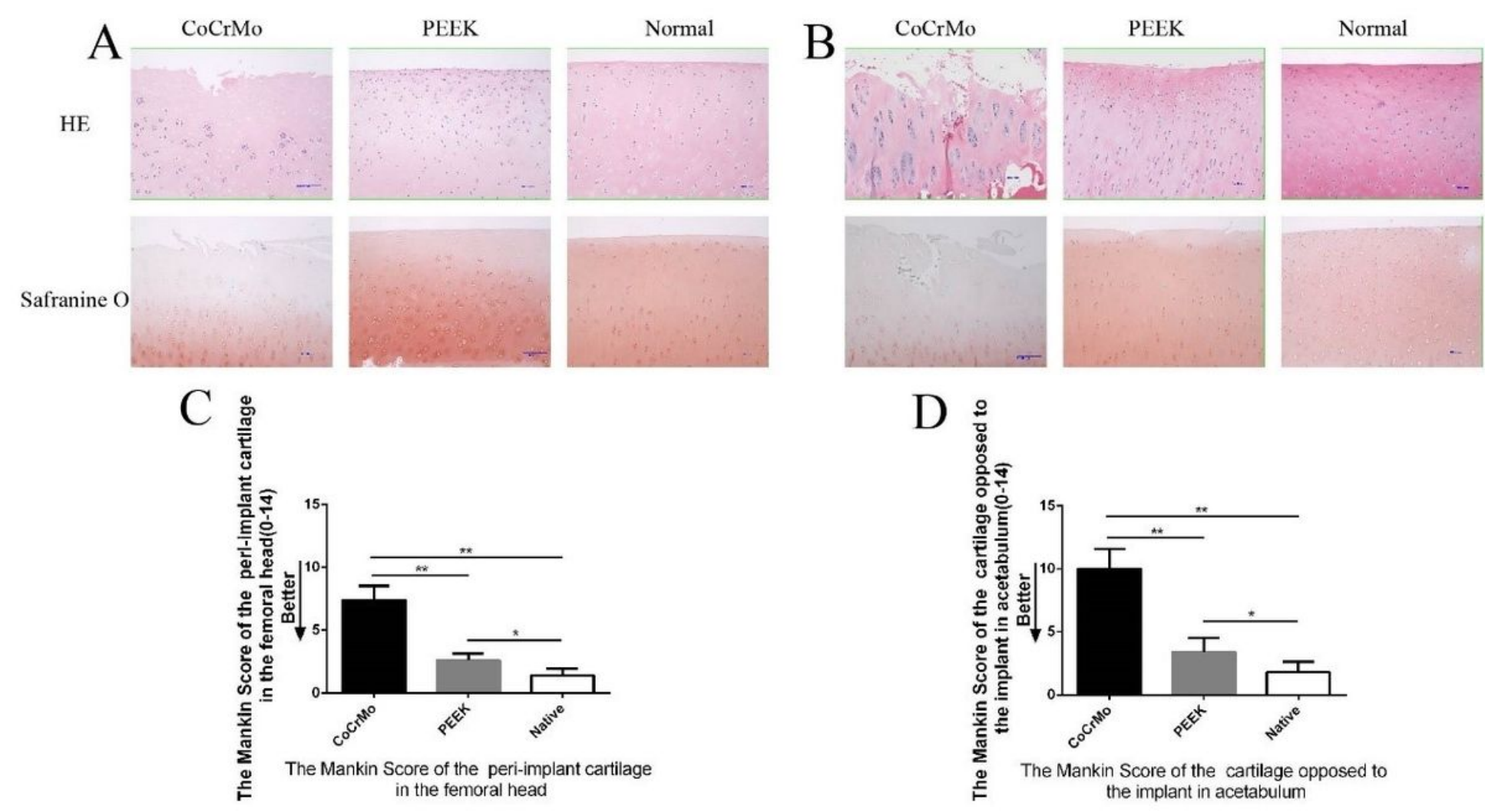

Figure 7

Histological analyses of the peri-implant cartilage in the femoral head and cartilage opposite to the implant in the acetabulum at 12 weeks after surgery. A. The peri-implant cartilage stained with hematoxylin and eosin (H\&E) and Safranin O. One native femoral head of a goat was used as a native control. B. The cartilage opposite to the implant in the acetabulum stained with H\&E and Safranin O. One native acetabulum of goat was used as a native control. C. Mankin scores for the peri-implant cartilage in the femoral head. $d$, Mankin scores of the cartilage opposite to the implant in the acetabulum. Data are expressed as mean \pm standard deviation $\left(n=5 ;{ }^{*}<<0.05\right.$, ** $\left.p<0.01\right)$. 

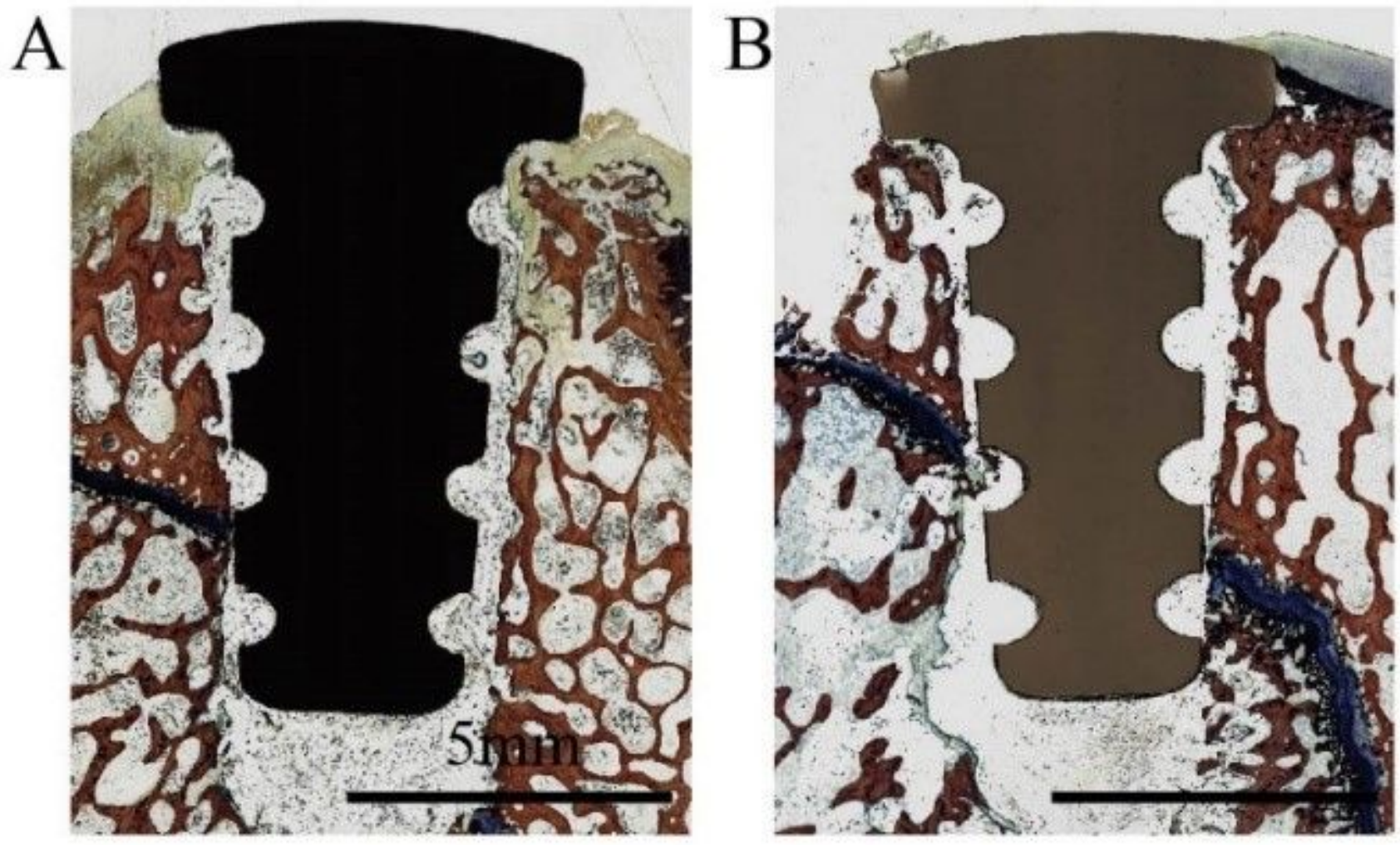

Figure 8

Van Gieson staining of the sections including an implant. A. Histological example of a CoCrMo implant 12 weeks after implantation. B. Histological example of a PEEK implant 12 weeks after implantation.

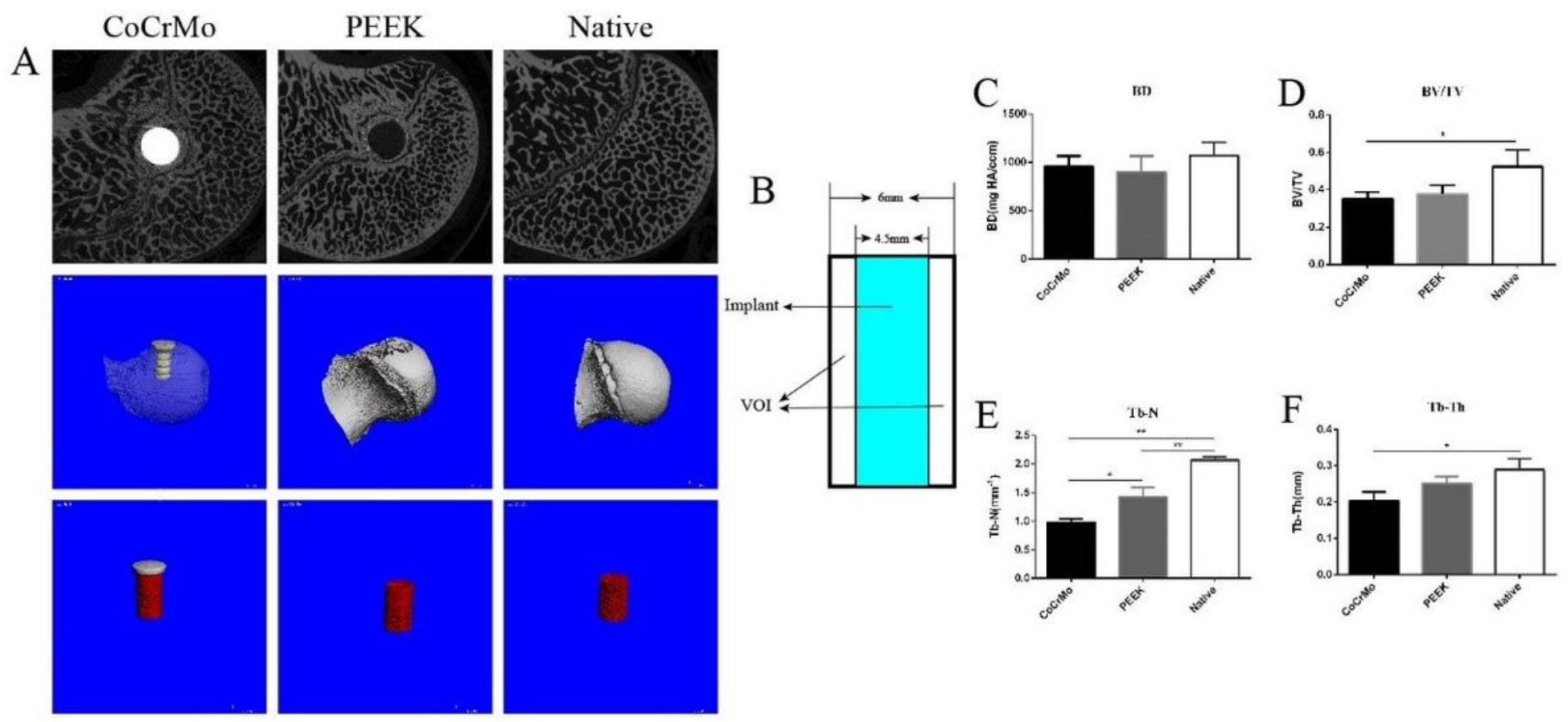

Figure 9 
Micro-CT of the femoral head with the implants. A. Radiological reconstruction images of the bone in the peripheral regions, 2D reconstructions of the femoral head are shown in the first row, 3D reconstructions of the femoral head are shown in the second row, and 3D reconstructions of the bone in the peripheral regions are shown in the third row. B. Diagrammatic sketch for volume of interests. C. Bone density (BD) in the peri-implant area. D. BV/CV of the peri-implant area. E. Trabecular number (Tb.N) in the peri-implant area. F. Trabecular thickness (Tb.Th) in the peri-implant area. The bars represent the mean, and the error bars represent the standard deviation. ${ }^{*} p<0.05,{ }^{*} \mathrm{p}<0.01$

A

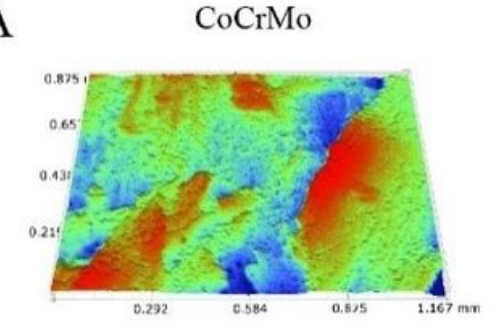

PEEK

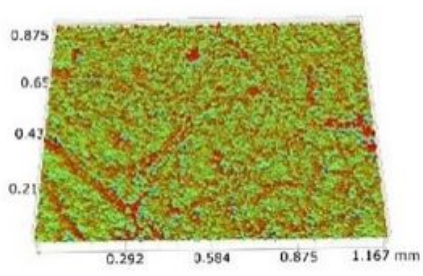

Native

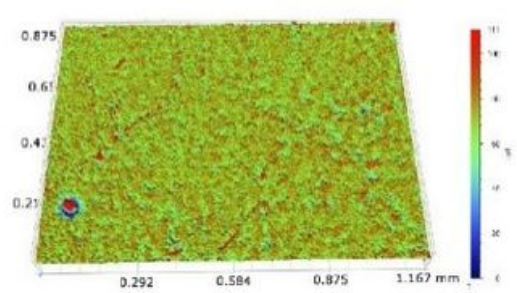

B

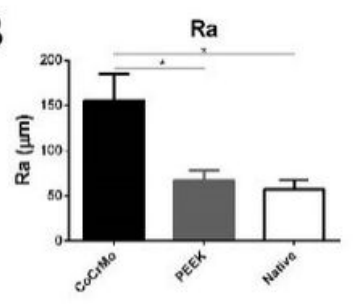

Figure 10

The surface roughness of the cartilage opposite to the implant in the acetabulum. A. 3D images using image analysis software of each sample in the CoCrMo, PEEK, and native group. B. The surface profile (Ra) was quantitatively analyzed. $\left(n=5,{ }^{*} p<0.05\right)$ CoCrMo, cobalt chromium molybdenum; PEEK, polyether ether ketone implant 